\title{
Long-range entanglement generation via frequent measurements
}

\author{
L.-A. Wu, D.A. Lidar, S. Schneider \\ Chemical Physics Theory Group, Chemistry Department, University of Toronto, \\ 80 St. George Street, Toronto, Ontario M5S 3H6, Canada
}

\begin{abstract}
A method is introduced whereby two non-interacting quantum subsystems, that each interact with a third subsystem, are entangled via repeated projective measurements of the state of the third subsystem. A variety of physical examples are presented. The method can be used to establish long range entanglement between distant parties in one parallel measurement step, thus obviating the need for entanglement swapping.
\end{abstract}

PACS numbers: 03.67.Lx, 03.65.Xp,74.20.Fg

\section{INTRODUCTION}

Entanglement is at the heart of quantum information processing 1], and is a resource that allows quantum processes to outperform their classical counterparts for tasks such as computation 2], communication [3], and cryptography [4]. The standard way to generate entanglement between distinguishable and initially separable particles is to let them interact directly for a certain amount of time: any non-trivial two-body Hamiltonian is capable of generating entanglement in this manner [5]. However, the direct interaction method poses limitations in the context of the generation of long-range entanglement, since in many systems the interaction strength typically decreases at least as fast as some power of the distance between particles. Here we introduce a different paradigm for entanglement generation: we show that it is possible to entangle two particles that never interact directly by means of repeated measurements of a third subsystem that interacts with both. In addition to its conceptual interest, we show that this scheme offers practical advantages for long-range entanglement generation.

We remark that other alternatives to the direct interaction method for entanglement generation are well known. E.g., in [6] linear optics is augmented by measurements to generate entanglement and perform quantum computation. Measurements of the phase of light transmitted through a cavity have also been proposed in 7] as a method to prepare entangled states and implement quantum computation in the case of atoms in optical cavities. Decoherence can also be used to create entanglement: e.g., atoms in a leaky cavity can become entangled conditioned on null detection at a photo detector placed outside the cavity [8, 9]. In Ref. [10] entanglement is generated in a similar manner conditioned instead upon detection.

Here we present a rather general theoretical framework for measurement-generated entanglement. Our scheme is inspired by the recent work by Nakazato, Takazawa, and Yuasa (NTY) 11], who investigated the effects of repeated rapid projective measurements on one subsystem of a bipartite system. Provided that the resulting operator on the unobserved subsystem has a non-degenerate largest eigenvalue, NTY showed that the unobserved sub- system is gradually projected into a pure state, independent of the initial state. This result leads to the question of whether a similar measurement scheme is capable of generating entanglement when the unobserved subsystem is itself multi-partite, non-interacting, and in an arbitrary initial state. We answer this question in the affirmative for a wide range of model systems, and establish general conditions for validity of the method. We note that an additional advantage of this measurement-based method for generating entanglement over the direct-interaction based method is that does not depend on sensitive timing of interactions: instead, entanglement is gradually purified as the number of measurements increases, and (under appropriate conditions) can be made arbitrarily high. An important conclusion from our study is that the method can be used to establish long range entanglement between distant particles, by measuring along a chain of intermediate particles. This provides an alternative to entanglement swapping that does not scale with the chain length. A similar result - long range entanglement from local (but non-repeated) measurements - was obtained in Ref. 12] for the ground state of an antiferromagnetic spin chain. We now turn a detailed description of our method.

\section{PRELIMINARIES}

NTY considered the following scenario: Consider a bipartite system composed of subsystems $A, B$, initially in the separable state $\rho_{A B}(0)=|\phi\rangle\langle\phi| \otimes \rho_{B}$, where $A$ is in a pure state $|\phi\rangle$ and $\rho_{B}$ is arbitrary. Subsystem $A$ is subject to projective measurements $P_{A}=|\phi\rangle\langle\phi|$ applied with period $\tau$. In between measurements the system evolves under the Hamiltonian

$$
H=H_{A}+H_{B}+H_{A B}
$$

(resp., the sum of free Hamiltonians and an interaction). It can be shown 11] that after $M$ such measurements the state of subsystem $B$, given that all outcomes were $|\phi\rangle$, is

$$
\rho_{B}(M \tau)=\frac{V_{B}(\tau)^{M} \rho_{B} V_{B}^{\dagger}(\tau)^{M}}{P_{M}}
$$


where $P_{M}$ is the survival probability, i.e., the probability of finding subsystem $A$ in its initial state,

$$
P_{M}=\operatorname{Tr}_{B}\left[V_{B}(\tau)^{M} \rho_{B} V_{B}^{\dagger}(\tau)^{M}\right]
$$

where $U=\exp (-i \tau H)$ (we use units where $\hbar=1$ ), and where

$$
V_{B}(\tau) \equiv\langle\phi|U| \phi\rangle
$$

is an operator on subsystem $B$ that is in general not Hermitian. However, one may still find left- and righteigenvectors with complex eigenvalues, whose modulus can be shown to be bounded between 0 and 1 . The central result of NTY is that in the limit of large $M$ and small but finite $\tau, \rho_{B}(M \tau)$ tends to a pure state independent of $B$ 's initial state. This result assumes that the largest eigenvalue $\lambda_{\max }$ of $V_{B}(\tau)$ is non-degenerate. The final pure state $\rho_{B}(M \tau)$ is then the corresponding right eigenvector $\left|u_{\max }\right\rangle$ and this outcome is found with probability

$$
P_{M} \rightarrow\left|\lambda_{\max }\right|^{2}\left\langle u_{\max } \mid u_{\max }\right\rangle\left\langle v_{\max }\left|\rho_{B}\right| v_{\max }\right\rangle,
$$

where $\left|v_{\max }\right\rangle$ is the corresponding left eigenvector. Note that because of $\tau$ 's finiteness the dynamics here is distinct from the Zeno effect and "quantum Zeno dynamics" 13 .

We now extend NTY's model by allowing $B$ itself to be composed of multiple non-interacting subsystems, $B=\left\{B_{1}, B_{2}, \ldots, B_{N}\right\}$, and pose the question: is the NTY measurement procedure capable of generating entanglement amongst $B$ 's subsystems, assuming that they all interact with the "station" $A$ ? The situation is illustrated in Fig. 1] To answer this questions we consider the following model: We set all internal Hamiltonians $H_{A}=H_{B_{i}}=0$, so that $H=H_{A B}$ (equivalently, we can always transform to an interaction picture rotating with the internal Hamiltonians; this will make $H_{A B}$ time dependent, but it will not introduce couplings between subsystems $B_{i}$ ). We assume that the $B$ subsystems are all qubits and the interaction between $A$ and all the $B_{i}$ is identical. We can then define total quasispin operators $\sigma_{\beta} \equiv \sum_{i=1}^{N} \sigma_{\beta}^{i}$ and write the interaction as $H_{A B}=\sum_{\alpha \in\{x, y, z\}} h_{\alpha} A_{\alpha} \otimes \sigma_{\alpha}\left(h_{\alpha}\right.$ real $)$ or, more simply,

$$
H_{A B}=\vec{A} \cdot \vec{\sigma}
$$

where the parameters $h_{\alpha}$ are included in the vector $\vec{A}$. We do not restrict subsystem $A$.

\section{GENERAL THEORY}

\section{A. A good basis}

We construct a basis that block-diagonalizes the effective post-measurement evolution operator $V_{B}(\tau)$. Let a basis for the $A$ subsystem be the measurement state $|\phi\rangle$

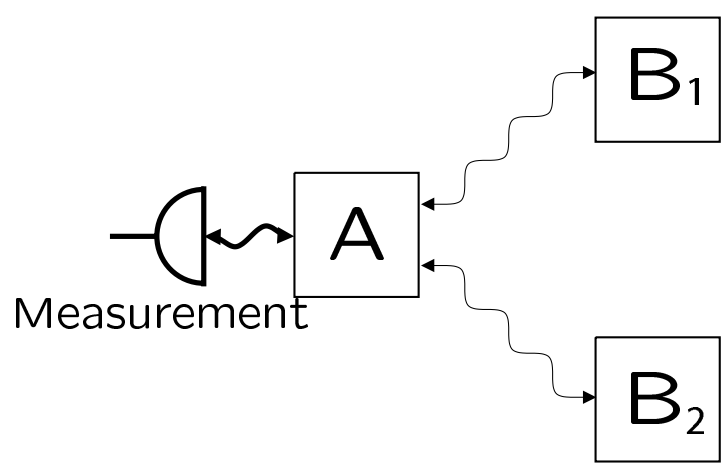

FIG. 1: Schematic illustration of our model: Systems $B_{1}$ and $B_{2}$ are coupled to system $A$; system $A$ can be measured with a projective measurement. Note that no direct coupling exists between systems $B_{1}$ and $B_{2}$.

and any set of states orthonormal to it, denoted $\left|\phi_{l}^{\perp}\right\rangle$, $l=1, \ldots, d-1$. Let $N$ be even and let a basis for the $B$ subsystem be constructed out of the usual spin basis $\left|S, M_{S}\right\rangle$, where $S$ is the total quasi-spin of the $N$ particles and $M_{S}$ its projection along the $z$-axis. We denote the (orthonormal) singlet states by $\left|s_{j}\right\rangle, j=1, \ldots, D_{N}=$ $N ! /[(N / 2+1) !(N / 2) !]$, and the remaining (orthonormal) states with $S>0$ by $\left|t_{k}\right\rangle, k=1, \ldots, K_{N}, K_{N}=2^{N}-D_{N}$.

Proposition 1: Consider the ordered basis $\left\{|\phi\rangle,\left|\phi_{1}^{\perp}\right\rangle, \ldots,\left|\phi_{d-1}^{\perp}\right\rangle\right\} \otimes\left\{\left|s_{1}\right\rangle, \ldots,\left|s_{D_{N}}\right\rangle,\left|t_{1}\right\rangle, \ldots,\left|t_{K_{N}}\right\rangle\right\}$. In this basis we have the block-diagonal representation

$$
V_{B}(\tau)=\left(\begin{array}{l|l}
I_{D_{N}} & \mathbf{0} \\
\hline \mathbf{0} & V_{B}^{s}
\end{array}\right)
$$

where $I_{D_{N}}$ is a $D_{N} \times D_{N}$-dimensional identity matrix. The maximal eigenvalue of $V_{B}(\tau)$ is 1 , and is at least $D_{N}$-fold degenerate.

Proof: The singlets states, $\left|S, M_{S}\right\rangle=|0,0\rangle$, are annihilated by the $\sigma_{\beta}$ when $N$ is even (since they are states with zero total quasi-spin). Therefore $H_{A B}\left|s_{j}\right\rangle=0$, independent of the state of subsystem $A$. In the ordered basis $H_{A B}$ is thus represented as

$$
H_{A B}=\left(\begin{array}{l|l}
\mathbf{0} & \mathbf{0} \\
\hline \mathbf{0} & H_{A B}^{\prime}
\end{array}\right)
$$

where the dimension of the upper-left block is $D_{N} \times D_{N}$ and that of the lower-right block is $K_{N} \times K_{N}$. Then

$$
U_{A B}=e^{-i \tau H_{A B}}=\left(\begin{array}{l|l}
I_{D_{N}} & \mathbf{0} \\
\hline \mathbf{0} & U_{A B}^{\prime}
\end{array}\right),
$$

where $U_{A B}^{\prime}=W^{\prime} e^{-i \tau \Lambda} W^{\prime \dagger}$ is unitary and $W^{\prime}$ is the unitary matrix that diagonalizes $H_{A B}^{\prime}: W^{\prime \dagger} H_{A B}^{\prime} W^{\prime}=\Lambda=$ $\operatorname{diag}\left(\lambda_{1}, \ldots, \lambda_{K_{N}}\right)$. Taking the expectation value wrt $|\phi\rangle$ we then find:

$$
V_{B}(\tau)=\sum_{j=1}^{D_{N}}\left|s_{j}\right\rangle\left\langle s_{j}\right|+V_{B}^{s},
$$

which is the claimed result, with $V_{B}^{s} \equiv$ $\sum_{k, k^{\prime}=1}^{K_{N}}\left|t_{k}\right\rangle\left(U_{A B}^{\prime}\right)_{\phi k, \phi k^{\prime}}\left\langle t_{k^{\prime}}\right|$ a $K_{N} \times K_{N}$-dimensional 
matrix. That the maximal eigenvalue is 1 follows from unitarity of $U_{A B}^{\prime}$ [1], and that it is at least $D_{N}$-fold degenerate is immediate from the representation (7). QED

Proposition 2 [11]: Non-degeneracy of $V_{B}(\tau)$ is a necessary condition for obtaining a pure state in the limit of large $M$.

Proof: In the degenerate case it follows from Eq. (2) that the method yields an equally weighted sum of degenerate pure states corresponding to the maximum eigenvalue of $V_{B}(\tau)$. QED

Note that $D_{2}=1$. Hence in the totally symmetric case we have been considering so far, for $N=2$ the method will generate a pure (maximally entangled) singlet state, provided $V_{B}^{s}$ has maximal eigenvalue with modulus smaller than 1. We give examples of corresponding Hamiltonians below. On the other hand, $D_{4}=2$ and hence due to degeneracy the method will not produce a pure entangled state for $N \geq 4$. However, we can still generate pure-state entanglement in the $N=4$ case by breaking the total permutation symmetry and only preserving the symmetry between 1,2 and 3,4 [e.g., having a coupling to subsystem $A$ such that $\sigma_{\beta}=a_{1}\left(\sigma_{\beta}^{1}+\sigma_{\beta}^{2}\right)+a_{2}\left(\sigma_{\beta}^{3}+\sigma_{\beta}^{4}\right)$, $\left.a_{1} \neq a_{2} \neq 0\right]$. We can then project out a one-dimensional subspace $|s\rangle_{12}|s\rangle_{34},|s\rangle_{i j}=\left(\left|0_{i} 1_{j}\right\rangle-\left|1_{i} 0_{j}\right\rangle\right) / \sqrt{2}$ is the singlet state, which is entangled for 1,2 and 3,4 but separable across the $12: 34$ partition. Similarly, for $N=2 n \geq 6$ and $\sigma_{\beta}=\sum_{i=1}^{n} a_{i}\left(\sigma_{\beta}^{2 i-1}+\sigma_{\beta}^{2 i}\right), a_{i} \neq a_{j} \neq 0$ the projected state is $|s\rangle_{12}|s\rangle_{34} \ldots . . .|s\rangle_{n-3, n-2}|s\rangle_{n-1, n}$.

\section{B. Invariance}

The results of the above discussion are invariant as long as the Hamiltonians belong to the family $\left\{U_{B} H_{A B} U_{B}^{\dagger}\right\}$, where $U_{B}$ is an arbitrary unitary transformation of the Hamiltonian of subsystem $B$. This enables the method to generate entangled states equivalent under local transformations. The invariance of $H_{A B}\left|s_{j}\right\rangle=0$ is $H_{A B}^{\prime}\left|s_{j}^{\prime}\right\rangle=0$, where $H_{A B}^{\prime}=U_{B} H_{A B} U_{B}^{\dagger}$ and $\left|s_{j}^{\prime}\right\rangle=U_{B}\left|s_{j}\right\rangle$. Thus, e.g., in the case of $N=2$, we can generate the other three Bell states by applying $U_{B}=X_{2}, Y_{2}, Z_{2}$ (where $X_{2}$ is the Pauli matrix $\sigma_{x}$ acting on subsystem $B_{2}$, etc.), which results, respectively, in $\left|s^{\prime}\right\rangle=\frac{1}{\sqrt{2}}(|11\rangle+|00\rangle), \frac{1}{\sqrt{2}}(|00\rangle-$ $|11\rangle), \frac{1}{\sqrt{2}}(|01\rangle+|10\rangle)$ as the outcome of the method.

\section{Degenerate maximal eigenvalue}

What happens when there are other states, either singlets or coming from $V_{B}^{s}$, that also have an eigenvalue with modulus 1? In this case NTY's result does not apply and entanglement may or may not be spoiled (though it is always reduced from maximally entangled). Two examples will illustrate: (i) $N=2$ : Suppose there is a triplet state $|00\rangle$ that also has $\left|\lambda_{\max }\right|=1$. Then the resulting state is the mixture $|s\rangle\langle s|+| 00\rangle\langle 00|$. This state is entangled as its partial transpose 14 has a negative eigenvalue of -0.207 . We will encounter this case in the Heisenberg model below. (ii) $N=4$ : Suppose the two singlet states $\left|s_{1}\right\rangle=|s\rangle_{12}|s\rangle_{34}$ and $\left|s_{2}\right\rangle=$ $\frac{1}{\sqrt{3}}\left[\left|t_{+}\right\rangle_{12}\left|t_{-}\right\rangle_{34}+\left|t_{-}\right\rangle_{12}\left|t_{+}\right\rangle_{34}-2\left|t_{0}\right\rangle_{12}\left|t_{0}\right\rangle_{34}\right]$ (where $\left|t_{\alpha}\right\rangle$ are triplets with projection quantum number $\alpha$ ) appear with eigenvalue one, but no other states do. With respect to the $12: 34$ cut $\left|s_{1}\right\rangle$ is a product state but $\left|s_{2}\right\rangle$ is clearly entangled. This state, just like in the previous example, has negative partial transpose, and we have entanglement across the $12: 34$ cut. These examples illustrate that degeneracy still allows for mixed state entanglement to be generated by our method. This is useful entanglement in the sense that it can be used for teleportation and all other QIP primitives 15.

\section{EXAMPLES}

We now discuss examples, first limiting ourselves to $A$ being a qubit and $N=2$. Our task then reduces to calculating the eigenvalues of the $3 \times 3$ matrix $V_{B}^{s}=\left\langle\phi\left|U_{A B}^{\prime}\right| \phi\right\rangle$. However, this requires diagonalization of $U_{A B}^{\prime}$, an $6 \times 6$ matrix, so cannot be done analytically in complete generality. Our basis for $A$ is $\left\{|\phi\rangle,\left|\phi^{\perp}\right\rangle\right\}$ and for $B$ is

$$
\begin{aligned}
\vec{\beta} \equiv\{|s\rangle & =\frac{1}{\sqrt{2}}(|01\rangle-|10\rangle),\left|t_{-}\right\rangle=|00\rangle, \\
\left|t_{0}\right\rangle & \left.\left.=\frac{1}{\sqrt{2}}(|01\rangle+|10\rangle),\left|t_{+}\right\rangle=|11\rangle\right\}\right\},
\end{aligned}
$$

without loss of generality (recall the invariance discussion above).

\section{A. Axial symmetry model}

Suppose $A_{z}=0$ and $A_{x}, A_{y}$ satisfy $\left[A_{x}, A_{y}^{2}\right]=$ $\left[A_{y}, A_{x}^{2}\right]=0$ (or $A_{x}$ or $\left.A_{y}=0\right)$, whence the Hamiltonian reads $H=\left[A_{x}\left(X_{1}+X_{2}\right)+A_{y}\left(Y_{1}+Y_{2}\right)\right] / 2$. We first consider as a special case the $X Y$ model: $A_{x}=J X$ and $A_{y}=J Y$, relevant for quantum information processing in solid state [16, 17, 18] and atomic [19] systems. It follows after some algebra that $H^{3}=|A|^{2} H$, where $|A|^{2}=A_{x}^{2}+A_{y}^{2}$. Therefore, the evolution is

$U_{A B}=e^{-i \tau H}=I-2\left(\frac{H}{|A|}\right)^{2} \sin ^{2} \frac{\tau|A|}{2}-i \frac{H}{|A|} \sin \tau|A|$,

which means that $V_{B}^{s}$ is a function only of $\langle\phi|H| \phi\rangle$ and $\left\langle\phi\left|H^{2}\right| \phi\right\rangle$. In particular, for the $X Y$ model we find, using $|A|=\sqrt{2} J$ 


$$
V_{B}(\tau)=\cos ^{2} \frac{\tau J}{\sqrt{2}}-\frac{X_{1} X_{2}+Y_{1} Y_{2}-\langle Z\rangle\left(Z_{1}+Z_{2}\right)}{2} \sin ^{2} \frac{\tau J}{\sqrt{2}}-i \frac{\langle X\rangle\left(X_{1}+X_{2}\right)+\langle Y\rangle\left(Y_{1}+Y_{2}\right)}{2 \sqrt{2}} \sin \sqrt{2} \tau J
$$

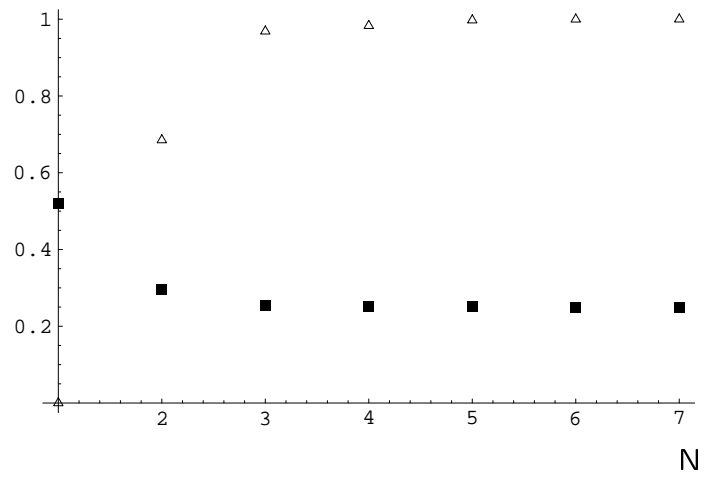

FIG. 2: Concurrence $(\triangle)$ and success probability $(\boldsymbol{\square}) P_{M}$ for $|\phi\rangle=\frac{1}{\sqrt{2}}(|0\rangle+|1\rangle)$ and $\tau J=\pi / 2$.

where $\langle X\rangle \equiv\langle\phi|X| \phi\rangle$, etc. It is simple to check that, as required from our general result, $V_{B}|s\rangle=|s\rangle$, i.e., the singlet state has eigenvalue 1 . Whether this eigenvalue is degenerate is now seen to depend on the measurement of the $A$ subsystem: If we choose $|\phi\rangle$ to be a $\sigma_{z}$-eigenstate then the additional eigenvalues of $V_{B}$ are found to be $\{1, \cos \sqrt{2} \tau J, \cos \sqrt{2} \tau J\}$, while if we choose $|\phi\rangle$ to be a $\sigma_{x}$ or $\sigma_{y}$-eigenstate then we find that the additional eigenvalues are $\left\{\cos ^{2} q, 1+3 \cos 2 q \pm \sqrt{\cos 4 q+\sin ^{4} q-1}\right\}$, where $q=\frac{\tau J}{\sqrt{2}}$. Thus, if we measure along $z$ then there is never pure state entanglement, but at times $\tau$ other than $n \pi / \sqrt{2} J$ we have a mixed entangled state, since the eigenstate corresponding to the additional eigenvalue 1 is found to be $|11\rangle$ (and recall the discussion above). In the case of measurement along $x$ or $y$ the eigenvalues periodically have modulus 1 , and with the exception of those times degenerary is avoided and we do obtain a pure entangled state (in particular, it is simple to show that this is true for all $\tau<\sqrt{2} \arccos (1 / 3) / J)$.

Next we consider the performance of the scheme after a finite number of steps (Fig. 2). To do so we calculate the concurrence [20] after $M$ steps of evolution/measurement for $J \tau=\pi / 2<\sqrt{2} \arccos (1 / 3)$, in the case of $|\phi\rangle$ a $\sigma_{x}$ eigenstate. In this case, as shown above, the singlet state is generated by the scheme. We also plot the survival probability $P_{M}$ as given by Eq. (3). After as few as three measurements the concurrence is essentially unity, indicating that the system is already in the desired singlet state. The scheme does, however, not work with unit probability: The probability $P_{M}$ of having projected system $A$ on the desired $\sigma_{x}$ eigenstate converges to $\sim 0.2$ for the values above, a value reached for $M=3$.

As another special case we consider a simplified form of the $d$-wave grain boundary qubit [21]: $H=\Delta\left(X_{1}+\right.$
$\left.X_{2}\right)+J Z\left(Z_{1}+Z_{2}\right)(\Delta$ is a tunneling parameter and $J$ is the Josephson coupling). Thus $\vec{A}=2(\Delta, 0, J Z)$, and we may use the general result (12), replacing $A_{y}$ by $A_{z}$. If we now choose $|\phi\rangle$ to be a $\sigma_{z}$-eigenstate then $V_{B}$ turns out to be unitary and hence all its eigenvalues have modulus one, and no entanglement is generated. However, if we choose $|\phi\rangle$ to be a $\sigma_{x}$-eigenstate, we find

$$
\begin{aligned}
V_{B}= & \cos ^{2} \phi-\sin ^{2} \phi\left(\cos ^{2} \theta X_{1} X_{2}+\sin ^{2} \theta Z_{1} Z_{2}\right) \\
& -\frac{i}{2} \sin 2 \phi \cos \theta\left(X_{1}+X_{2}\right)
\end{aligned}
$$

where $\phi=\tau \sqrt{\Delta^{2}+J^{2}}$ and $\tan \theta=J / \Delta$. It is again easy to check that $V_{B}|s\rangle=|s\rangle$. One of the other three eigenvalues is $1-2 \sin ^{2} \phi \sin ^{2} \theta$. The other two are complex conjugates and for short times $\tau$ such that $\tan ^{2} \phi<4 \cos ^{2} \theta / \sin ^{4} \theta$ have the same amplitude (1 $\left.2 \sin ^{2} \phi \sin ^{2} \theta\right)^{1 / 2}$. For instance, when $\theta=\pi / 4$, the amplitudes of the three eigenvalues are $\cos ^{2} \phi, \cos \phi, \cos \phi$, which vanish rapidly when raised to the power of the number of measurements.

Finally, note that the results presented so far are not restricted to $A$ being a qubit: the unitary operator (12) is valid even when $A$ is a multi-level system, as long as the condition $\left[A_{x}, A_{y}^{2}\right]=\left[A_{y}, A_{x}^{2}\right]=0$ is satisfied.

\section{B. Heisenberg model}

We now consider the Heisenberg interaction $H=$ $J \vec{\sigma} \cdot\left(\vec{\sigma}_{1}+\vec{\sigma}_{2}\right)$. After showing that $\left(H+J \vec{\sigma}_{1} \cdot \vec{\sigma}_{2}\right)^{2}=$ $9 J^{2}$ and $\left[H, J \vec{\sigma}_{1} \cdot \vec{\sigma}_{2}\right]=0$, it is simple to compute $e^{-i \tau\left(H+J \vec{\sigma}_{1} \cdot \vec{\sigma}_{2}\right)}$, from which we directly obtain

$$
\begin{aligned}
U_{A B}= & e^{-i \tau H}=e^{i \tau J \vec{\sigma}_{1} \cdot \vec{\sigma}_{2}}\{\cos (3 \tau J) \\
& \left.-i \sin (3 \tau J)\left[\vec{\sigma} \cdot\left(\vec{\sigma}_{1}+\vec{\sigma}_{2}\right)+\vec{\sigma}_{1} \cdot \vec{\sigma}_{2}\right] / 3\right\} .
\end{aligned}
$$

$V_{B}$ then is identical to $U_{A B}$ provided one replaces with $\langle\phi|\vec{\sigma}| \phi\rangle$ the operator $\vec{\sigma}$ in $U_{A B}$. The four eigenvalues are $\left\{1, e^{-2 i \tau J}, e^{i \tau J}\left(\cos 3 \tau J \pm i \frac{1}{3} \sin 3 \tau J\right)\right\}$, the last two having the same amplitude $1-\frac{8}{9} \sin ^{2} 3 \tau J<1$. The corresponding eigenvectors are, respectively, $\vec{\beta}$ [Eq. (11)]. Since the first two eigenvalues both have magnitude 1 , we have the case discussed above: a pure entangled state cannot be projected out by our method, but we can prepare a mixed entangled state that is useful for all QIP protocols. Note further that these Heisenberg model results hold for the entire class of Hamiltonians $H=\vec{A}_{\sigma} \cdot\left(\vec{\sigma}_{1}+\vec{\sigma}_{2}\right)$, when $\vec{A}_{\sigma}$ is generated from an arbitrary 2-dimensional unitary transformation $U_{A}, \vec{A}_{\sigma}=U_{A} J \vec{\sigma} U_{A}^{\dagger}$ (this is 
different from the invariance under a rotation of the $B$ system considered above).

\section{Bosonic media}

We now consider a photon or phonon $(A)$ interacting symmetrically with two identical qubits $(B)$. The JaynesCummings Hamiltonian is

$$
H=\epsilon b^{\dagger} b+g\left(\sigma_{1}^{z}+\sigma_{2}^{z}\right)+\frac{J}{2}\left[b\left(\sigma_{1}^{+}+\sigma_{2}^{+}\right)+b^{\dagger}\left(\sigma_{1}^{-}+\sigma_{2}^{-}\right)\right]
$$

where $b$ is a bosonic annihilation operator, and whence $\vec{A}=\left(J\left(b+b^{\dagger}\right), i J\left(b-b^{\dagger}\right), g\right)$. Such a model can easily be realized using microwave cavity QED 22! (two atoms in one or two cavities) or trapped ions [23]. We can exactly diagonalize $H$ by writing it as a direct sum over three-dimensional matrices in the basis $\left|n-1, t_{+}\right\rangle,\left|n, t_{0}\right\rangle$ and $\left|n+1, t_{-}\right\rangle$, where $|n\rangle$ are number states and $\left|t_{\alpha}\right\rangle$ are triplet states of the two qubits. Note that $\mathcal{N} \equiv\left[b^{\dagger} b+\right.$ $\left.\left(\sigma_{1}^{z}+\sigma_{2}^{z}\right)\right]$ is a conserved quantity and hence $H$ is block diagonal in its eigenvalues. For simplicity we consider the case with $g=\epsilon$ and measure the single photon state $|\phi\rangle=b^{\dagger}|0\rangle=|1\rangle$ (this projects onto a single block of the infinite matrix $\left.U_{A B}\right)$. In this case we readily find the already diagonal

$$
\begin{gathered}
V_{B}(\tau)=\operatorname{diag}\left\{1, e^{-2 i \epsilon \tau} \frac{3+2 \cos (\sqrt{10} \tau J)}{5}\right. \\
\left.e^{-i \epsilon \tau} \cos (\sqrt{6} \tau J), \cos (\sqrt{2} \tau J)\right\}
\end{gathered}
$$

in the ordered basis $\left\{|1\rangle|s\rangle,\left|1, t_{+}\right\rangle,\left|1, t_{0}\right\rangle,\left|1, t_{-}\right\rangle\right\}$. Thus, as long as we make sure that $\tau<2 \pi / \sqrt{10} J$ the method will project out a pure singlet state. It follows from our discussion of invariance above that another Bell state $\frac{1}{\sqrt{2}}(|01\rangle+|10\rangle)$ can be projected out if the two qubits couple to the photon or phonon with opposite signs.

\section{Multilevel Systems}

Let us now consider a rather general, though abstract multilevel case. Assume that subsystem $B$ consists of two particles that have $M \geq 2$ levels each, and that the interaction Hamiltonian with subsystem $A$ (also an $M$ level system) is of the form

$$
H=\sum_{i, j=1}^{M} A_{i j}\left(O_{i j}^{1}+O_{j i}^{2}\right),
$$

where $O_{i j}=E_{i j}-E_{j i}$ and $E_{i j}=|i\rangle\langle j|$ is a matrix whose elements are zero everywhere except for a 1 at position $(i, j)$. Namely, there is an $S O(M)$ symmetry for odd $M$ or an $S p(M)$ symmetry for even $M$. In this case $H$ has a single non-degenerate eigenvector with zero eigenvalue. This state is entangled. For instance, when $N=3$, we have two qutrits, which can be represented in the spherical basis $\{|-1\rangle,|0\rangle,|+1\rangle\}$. The state with zero eigenvalue is

$$
|\Psi\rangle=\left(|1\rangle_{1}|-1\rangle_{2}-|0\rangle_{1}|0\rangle_{2}+|-1\rangle_{1}|1\rangle_{2}\right) / \sqrt{3}
$$

and is maximally entangled. In general, the onedimensional subspace containing the state with zero eigenvalue is the irreducible representation $(0,0, \ldots, 0)$ of $S O(M)$ or $S p(M)$. This state is the generalization of the singlet state that arose in the general theory and examples treated above when the subsystems were qubits.

\section{PERTURBATIVE TREATMENT}

It is apparent from the above examples that the success of our method depends on keeping the period $\tau$ between two measurements short. For instance, in the bosonic example, we require $\tau<2 \pi / \sqrt{10} J$ in order to prevent the appearance of another eigenvalue with amplitude one. Let us therefore consider a short-time expansion of $V_{B}(\tau)$. To first order

$$
\begin{aligned}
& V_{B}(\tau)^{M}=\left(I-i \tau\left\langle\phi\left|H_{A B}\right| \phi\right\rangle\right)^{M} \\
& \stackrel{M \rightarrow \infty}{\longrightarrow} \exp \left(-i t\left\langle\phi\left|H_{A B}\right| \phi\right\rangle\right)
\end{aligned}
$$

where $M \tau=t$ (constant), i.e., the evolution is unitary. This is the quantum Zeno effect, which completely decouples the interaction among all parties of subsystem $B$, so that no entanglement can be generated in this limit. Thus the dominant contribution to entanglement generation originates from the second-order term, which contains the self-correlation of subsystem $B$. Consider for simplicity the case $\left\langle\phi\left|H_{A B}\right| \phi\right\rangle=0$ (as in our last example). Letting $M \tau^{2} / 2=t^{2}$ (constant), we have

$$
V_{B}(\tau)^{M} \stackrel{M \rightarrow \infty}{\longrightarrow} e^{-t^{2}\left\langle\phi\left|H_{A B}^{2}\right| \phi\right\rangle}
$$

All eigenstates of $\left\langle\phi\left|H_{A B}^{2}\right| \phi\right\rangle$ with nonzero eigenvalues are rapidly suppresed as $t$ (in practice $M$ ) increases, while those with eigenvalue zero survive. Since it is much simpler to analytically calculate $\left\langle\phi\left|H_{A B}^{2}\right| \phi\right\rangle$ than $V_{B}(\tau)$, this perturbative method provides a relatively simple tool for estimating the possibility of entanglement generation via our method, for complicated systems. We consider spin-orbital coupling as another illustrative example: $H=h L_{x}\left(X_{1}+X_{2}\right)+h L_{y}\left(Y_{1}+Y_{2}\right)$, denoting two spin qubits that couple with the same orbital angular momentum. If we measure the eigenstate $|\phi\rangle=|l, 0\rangle$ of the orbital component, we find $\langle\phi|H| \phi\rangle=0$ and $\left\langle\phi\left|H^{2}\right| \phi\right\rangle=h l(l+1)\left(2+X_{1} X_{2}+Y_{1} Y_{2}\right)$. The eigenvalues are $h l(l+1)\{0,2,4,2\}$ with respective eigenvectors $\vec{\beta}$, meaning that the singlet state $|s\rangle$ is projected out in the second-order analysis. Note that this example is qualitatively different from the previous one since subsystem $A$ here does not refer to a physically separate particle. 


\section{PREPARATION OF LONG-DISTANCE ENTANGLEMENT}

Finally we come to our main result: the generation of long-distance bi-partite entanglement. Since in our discussion above there was no restriction on the size of the $A$ subsystem, in a sense long-distance entanglement generation already follows from the results above. However, it is important to specify how the measurements on $A$ can be carried out. Assume that the $A$ subsystem is composed of $N-2$ qubits $2,3, \ldots, N-1$ and we wish to entangle the two $B$-subsystem qubits $1, N$. We consider again as an illustrative example a simplified form of the $d$-wave grain boundary qubit [21]:

$$
U_{A B}=\exp \left[-i J \tau\left(X_{1}+X_{N}\right)-i \Delta \tau\left(Z_{1} Z_{2}+Z_{2} Z_{3}+h_{3}+Z_{N-2} Z_{N-1}+Z_{N-1} Z_{N}\right)\right]
$$

where $h_{3}=\sum_{i=3}^{N-3} Z_{i} Z_{i+1}$. We choose $|\phi\rangle$ to be the state $|+\rangle_{2}|R\rangle|+\rangle_{N-1}$, where $|R\rangle=|+\rangle_{3}|+\rangle_{4} \ldots|+\rangle_{N-2}$, where $|+\rangle=(|0\rangle+|1\rangle) / \sqrt{2}$. It follows after some calculations that

$$
\begin{aligned}
V_{B}= & \frac{1}{2}\left\{a \left[\cos ^{2} \phi-\sin ^{2} \phi\left(\cos ^{2} \theta X_{1} X_{N}+\sin ^{2} \theta Z_{1} Z_{N}\right)-i \frac{1}{2} \sin 2 \phi \cos \theta\left(X_{1}+X_{N}\right)\right.\right. \\
& +b\left[\cos ^{2} \phi-\sin ^{2} \phi\left(\cos ^{2} \theta X_{1} X_{N}-\sin ^{2} \theta Z_{1} Z_{N}\right)-i \frac{1}{2} \sin 2 \phi \cos \theta\left(X_{1}+X_{N}\right)\right\}
\end{aligned}
$$

where

$$
\begin{aligned}
a & =\left\langle R\left|e^{-i \Delta \tau\left(Z_{3}+Z_{N-2}+h_{3}\right)}\right| R\right\rangle=\left\langle R\left|e^{-i \Delta \tau\left(-Z_{3}-Z_{N-2}+h_{3}\right)}\right| R\right\rangle \\
b & =\left\langle R\left|e^{-i \Delta \tau\left(Z_{3}-Z_{N-2}+h_{3}\right)}\right| R\right\rangle=\left\langle R\left|e^{-i \Delta \tau\left(-Z_{3}+Z_{N-2}+h_{3}\right)}\right| R\right\rangle
\end{aligned}
$$

(and are real numbers for odd $N), \phi=\tau \sqrt{J^{2}+\Delta^{2}}$, $\tan \theta=\Delta / J$. Two of the eigenvalues and eigenstates are:

$E_{1}=\frac{1}{2}\left\{a+b\left(1-2 \sin ^{2} \phi \sin ^{2} \theta\right)\right\} ; \frac{1}{\sqrt{2}}\left(\left|1_{1} 0_{N}\right\rangle-\left|0_{1} 1_{N}\right\rangle\right)$,
$E_{2}=\frac{1}{2}\left\{b+a\left(1-2 \sin ^{2} \phi \sin ^{2} \theta\right)\right\} ; \frac{1}{\sqrt{2}}\left(\left|0_{1} 0_{N}\right\rangle-\left|1_{1} 1_{N}\right\rangle\right)$.

Thus, as desired, entanglement is generated between the $B$ subsystem particles $1, N$. Note that since the measurement of all the particles of the $A$ subsystem is carried out in parallel this method for long-range entanglement generation is independent of the distance $N$.

The amplitudes of the other two eigenvalues are $\sqrt{E_{1} E_{2}}$ in the case of odd $N \geq 5$. Since $\max \left(\left|E_{2}\right|,\left|E_{1}\right|\right)>$ $\sqrt{E_{1} E_{2}}$ for sufficiently short time, and $E_{1}-E_{2}=$ $2(\sin \phi)^{2}(\sin \theta)^{2}(a-b)$ as long as $a \neq b$, the procedure prepares either $\frac{1}{\sqrt{2}}\left(\left|1_{1} 0_{N}\right\rangle-\left|0_{1} 1_{N}\right\rangle\right)$ or $\frac{1}{\sqrt{2}}\left(\left|0_{1} 0_{N}\right\rangle-\right.$ $\left.\left|1_{1} 1_{N}\right\rangle\right)$. We find, e.g, for $N=5: a=\cos 2 \Delta \tau, b=1$ and $\frac{1}{\sqrt{2}}(|00\rangle-|11\rangle)$ is projected out, while for $N=7$ : $a=\frac{1}{4}(3+\cos 4 \Delta \tau), b=\cos 2 \Delta \tau$ and $\frac{1}{\sqrt{2}}(|01\rangle-|10\rangle)$ is projected out. The case of even $N$ is more complicated since the amplitudes of other two eigenvalues depend on the values of $a$ and $b$. E.g., in the $N=4, V_{B}$ has the same form as above but $a=e^{-i \Delta \tau}$ and $b=e^{i \Delta \tau}$; all roots have the same amplitude and no pure state will be projected out.

\section{CONCLUSIONS}

We have introduced a measurement-based method for entangling two systems that only interact indirectly, via a third system. Applications range from solid state to atomic quantum information processing. One of the advantages of this measurement-based method of entanglement generation is that it does not depend on precisely timed interactions, as are interactions-based schemes. The method can be used to prepare arbitrarily longdistance entanglement via a chain of intermediate particle in one parallel measurement step. This may have useful applications in reducing the latency overhead in quantum communication in quantum computing architectures. An interesting open question which we leave for future research is whether the same method can be used to apply quantum logic gates between non-interacting particles.

Acknowledgents.- D.A.L. acknowledges support from the Sloan Foundation, D-Wave Systems, Inc., and the DARPA-QuIST program (managed by AFOSR under agreement No. F49620-01-1-0468).
[1] J. Gruska, Quantum Computing (Mc Graw-Hill, London, 1999).
[2] A. Ekert and R. Jozsa, Phil. Trans. Roy. Soc. (Lond.) 356, 1769 (1998). 
[3] R. Raz, in Proceedings of 31st Annual ACM Symposium on Theory of Computing (STOC) (ACM, New York, NY, 1999), p. 358.

[4] A. Ekert, Phys. Rev. Lett. 67, 661 (1991).

[5] J. Zhang, J. Vala, S. Sastry, K.B. Whaley, Phys. Rev. A 67, 042313 (2003).

[6] E. Knill, R. Laflamme, and G.J. Milburn, Nature 409, 46 (2001).

[7] A. Sørensen and K. Mølmer, Phys. Rev. Lett. 91, 097905 (2003).

[8] M.B. Plenio, S.F. Huelga, A. Beige and P.L. Knight, Phys. Rev. A 59, 2468 (1999).

[9] A. Beige, S. Bose, D. Braun, S.F. Huelga, P.L. Knight, M.B. Plenio, V.Vedral, J. Mod. Optics 47, 2583 (2000).

[10] C. Cabrillo, J.I. Cirac, P. Garcia-Fernández, P. Zoller, Phys. Rev. A 59, 1025 (1999).

[11] H. Nakazato, T. Takazawa, K. Yuasa, Phys. Rev. Lett. 90, 060401 (2003).

[12] F. Verstraete, M.A. Martin-Delgado, J.I. Cirac, Phys. Rev. Lett. 92, 087201 (2004).

[13] P. Facchi and S. Pascazio, Phys. Rev. Lett. 89, 080401 (2002).
[14] A. Peres, Phys. Rev. Lett. 77, 1413 (1996).

[15] M. Horodecki, P. Horodecki, R. Horodecki, Phys. Rev. Lett. 78, 574 (1997).

[16] D. Mozyrsky, V. Privman, and M.L. Glasser, Phys. Rev. Lett. 86, 5112 (2001).

[17] A. Imamoḡlu, D.D. Awschalom, G. Burkard, D.P. DiVincenzo, D. Loss, M. Sherwin and A. Small, Phys. Rev. Lett. 83, 4204 (1999).

[18] L. Quiroga and N.F. Johnson, Phys. Rev. Lett. 83, 2270 (1999).

[19] S.-B. Zheng and G.-C Guo, Phys. Rev. Lett. 85, 2392 (2000).

[20] W.K. Wootters, Phys. Rev. Lett. 80, 2245 (1998).

[21] A. Blais and A.M. Zagoskin, Phys. Rev. A 61, 042308 (2000).

[22] A. Rauschenbeutel, G. Nogues, S. Osnaghi, P. Bertet, M. Brune, J.M. Raimond, and S. Haroche, Phys. Rev. Lett. 83, 5166 (1999).

[23] D. J. Wineland, C. Monroe, W. M. Itano, D. Leibfried, B. E. King, and D. M. Meekhof, J. of Res. of the National Inst. of Standards and Technology 103, 259 (1998). 